\title{
The effect of adhesive strength of hydroxyapatite coating on the stability of hydroxyapatite-coated prostheses in vivo at the early stage of implantation
}

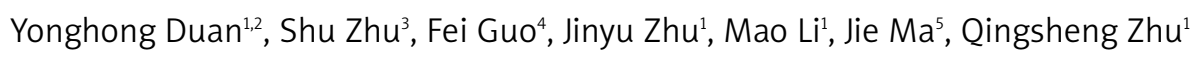

\author{
${ }^{1}$ Orthopedic Institute of Chinese People's Liberation Army, Xijing Hospital, Fourth \\ Military Medical University, Xi'an, Shanxi, China \\ 2Department of Orthopaedic Surgery, 451 Hospital of Chinese People's Liberation \\ Army, Xi'an, Shanxi, China \\ 3Department of Dentistry, Chinese People's Liberation Army, Fourth Military Medical \\ University, Xi'an, Shanxi, China \\ 4Department of Orthopaedic Surgery, Linton Sanitarium of Chinese People's \\ Liberation Army, Xi'an, Shanxi, China \\ ${ }^{5}$ Department of Neurosurgery, Tangdu Hospital, Fourth Military Medical University, \\ Xi'an, Shanxi, China
}

Submitted: 12 January 2011

Accepted: 11 June 2011

Arch Med Sci 2012; 8, 2: 199-208

DOI: 10.5114/aoms.2012.28545

Copyright ( 2012 Termedia \& Banach

\begin{abstract}
Introduction: With the increase in joint revision surgery after arthroplasty, defects of hydroxyapatite (HA)-coated prostheses have been observed increasingly often. These defects adversely affect the prosthetic stability in vivo. This study has analyzed the potential effect of the adhesive strength of HA coating on the stability of HA-coated prostheses in vivo after its implantation.

Material and methods: Sixty experimental rabbits were divided into HA- and Ticoated groups. HA-coated prostheses were implanted into the bilateral epicondyle of rabbits femurs. Ti-coated prostheses were implanted as control. At different time points (4, 9, and 15 weeks) after implantation, bone tissue samples were fetched out respectively for histomorphometric analysis. Push-out testing was used to detect the ultimate shear strength at the bone-prosthesis interface. Scanning electron microscope (SEM) observation and energy-dispersive $\mathrm{X}$-ray spectroscopy (EDX) analysis were used to observe the changes in surface composition of the prostheses after the ultimate shear strength testing. The coating adhesive strength of two kinds of coatings were also examined by scratch testing.

Results: Hydroxyapatite coating has an obvious advantage in facilitating osteogenesis and its plays a critical role in the stability of prostheses. However, the ultimate shear strength of HA-coated prostheses is much lower than that of Ticoated implants $(p<0.01)$. Further study has demonstrated that the stability of HA-coated prostheses in vivo is affected by the relatively low adhesive strength between coating and substrate.

Conclusions: Obvious advantage in facilitating osteogenesis around HA-coated prostheses is not the only factor that determines the stability of prostheses in vivo.
\end{abstract}

Key words: hydroxyapatite coating, artificial joints, stability, biomechanical, adhesive strength.

\section{Introduction}

Hydroxyapatite (HA)-coated artificial joints and dental implants have been gradually applied and promoted in the clinic because of their effective bone conduction and bone induction. The satisfactory effect of HA-coated prostheses in bone ingrowth and early stability has been widely recognized

\author{
Corresponding author: \\ Dr. Qingsheng Zhu \\ Orthopedic Institute \\ of Chinese People's \\ Liberation Army \\ Xijing Hospital \\ Fourth Military Medical \\ University \\ Xi'an, Shanxi 710032 \\ People's Republic of China \\ Phone: +86 2984775277 \\ E-mail: zhuqsh@126.com
}


by doctors and investigators [1-3]. It is reported that HA-coated prostheses induce rapid attached growth and ingrowth of the bone, increase the rate and amount of bone formation around the implant, reduce the risk of periprosthetic fibrosis and the period of postoperative recovery, and play an active role in the biological fixation and prosthetic stability at the early stage of prosthesis implantation [4-7].

However, with the increase in joint revision surgery after arthroplasty, defects of HA-coated prostheses, the decohesion and degradation of HA coating, and the wear of prostheses caused by $\mathrm{HA}$ particles have been observed gradually more often. These defects adversely affect the prosthetic stability in vivo [8-12]. In addition, HA coating's decohesion and fatigue fracture during the surgical procedure and under postoperative loading exacerbate the gap formation at prosthesis-coating and boneprosthesis interfaces and facilitate canal formation for HA particle migration, which accelerates the prosthetic loosening in vivo [12]. This study has further investigated the effects of adhesive strength of HA coating on the stability of HA-coated prostheses in vivo by histopathological observation, biomechanical testing, energy-dispersive $X$-ray spectroscopy (EDX) analysis, and testing of the coating adhesive strength.

\section{Material and methods}

\section{Material preparation}

Ti-6Al-4V medical metal substrate was sandblasted and plasma-sprayed to make the HA-coat-
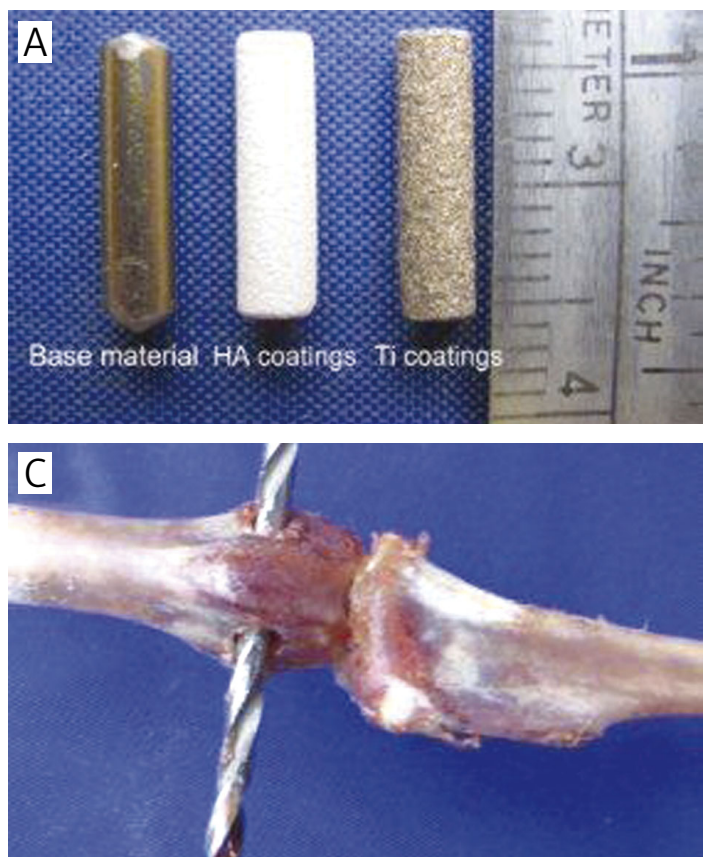

Figure 1. Experimental materials for plasma spraying and diagram of implantation surgery. A - Ti alloy substrate and HA-, Ti-coated prostheses; B - specimens for testing the characteristics of substrate and different coatings;

C, D - the site for prosthesis implantation and the surgical procedures ed prostheses and the samples for testing of coating adhesive strength with about $30 \%$ surface porosity. Ti-coated material with the same specifications was used as control. The prostheses have the following parameters: $\Phi=3 \mathrm{~mm} \times 12 \mathrm{~mm}$, coating thickness about $150 \mu \mathrm{m}$. The samples for testing of coating adhesive strength have the following parameters: $\Phi=20 \mathrm{~mm} \times 3 \mathrm{~mm}$, coating thickness about $40 \mu \mathrm{m}$. All the coating processing was done by Jinghang Institute for Biomaterials, Beijing, China. The prostheses and the samples for testing of coating adhesive strength can be seen in Figures $1 \mathrm{~A}$ and $\mathrm{B}$.

\section{Experimental animals and implantation}

Sixty male New Zealand white rabbits (body weight: $2.8-3.0 \mathrm{~kg}$; age: 6 months) were used in this experiment. They were provided by the Experimental Animal Center, Fourth Military Medical University, China, and the study was approved by the Ethics Committee of this University. All experimental animals were divided into $\mathrm{HA}$ - and Ti-coated groups with 30 animals in each group. Rabbits were anesthetized with $2 \%$ pentobarbital injection (40 mg/kg) through the marginal ear vein. The operation was performed under aseptic conditions. A $1.5 \mathrm{~cm}$ longitudinal incision was made on the lateral knee joint; one hole was drilled on the bilateral epicondyle of the femurs behind the lateral edge of the femoral trochlea and toward the medial epicondyle of the femur with a $\Phi=3.2 \mathrm{~mm}$ drill. After the prostheses were placed into the hole, the incisions were closed with sutures. Each animal received daily intramuscular injection of penicillin
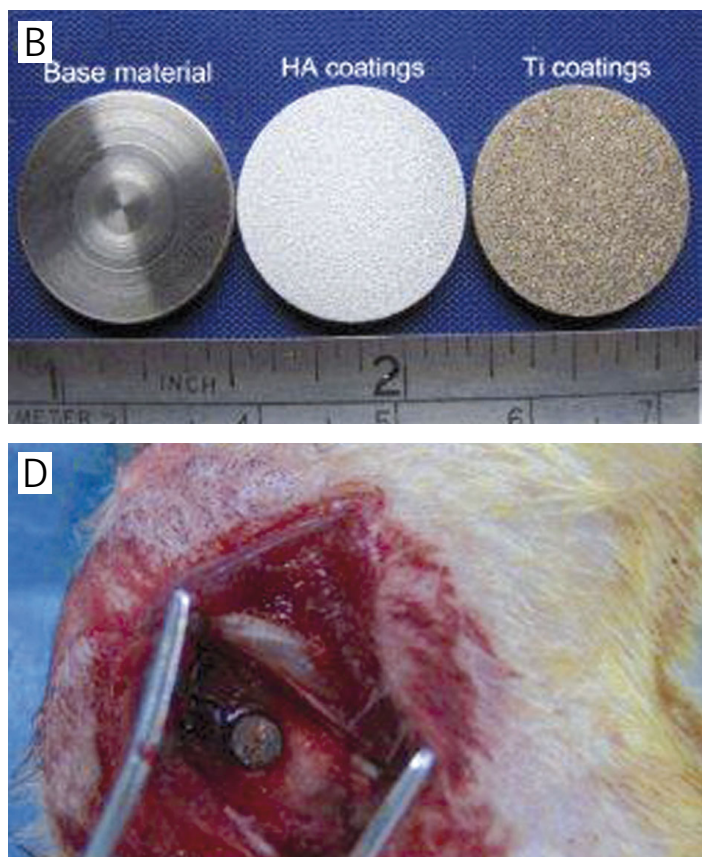
400,000 units to avoid wound infection during the first week after surgery. Ten animals from each group were killed respectively 4, 9, and 15 weeks after the surgery. Bone tissue samples which contained prostheses were fixed with $90 \%$ ethanol for further investigations. Figures $1 \mathrm{C}$ and D illustrate the site of prostheses implantation and the actual surgical procedures respectively.

\section{Testing of coating adhesive strength}

The testing of coating adhesive strength was carried out on $40 \mu \mathrm{m}$ thickness HA- or Ti-coated samples with WS-2005 coating adhesion automatic scratch tester (LICTP.AC, China; scratch head movement: $2 \mathrm{~mm} / \mathrm{min}$; maximum load: $100 \mathrm{~N}$; loading rate: $20 \mathrm{~N} / \mathrm{min}$ ).

\section{Histopathological evaluation}

The bone ingrowth on the surface of implanted prostheses and the morphology of the prosthesisbone interface were observed through histological staining of the sections.

\section{Improved Ponceau trichrome staining}

Specimens were cut and harvested immediately after killing the animals and were fixed in $90 \%$ ethanol for 1 week. Then specimens were treated with liquid plastic gradient to make plastic blocks that contained the metal implants; the blocks were then sectioned using a Leica SM2500 hard tissue microtome to obtain $7 \mu \mathrm{m}$ thickness sections. The hard tissue sections were stained with Ponceau trichrome stain according to the following procedure: 1) preparation of the staining solution: a) picric acid-orange mixture ( $1 \%$ orange $G$ solution and saturated picric acid solution were mixed at $1: 9$ ratio); b) Ponceau mixture (2\% R2 Ponceau in 1\% acetic acid solution and $2 \%$ crystal Ponceau in $1 \%$ acetic acid solution were mixed at $4: 1$ ratio); 2 ) the staining procedure: a) slides were incubated in $1 \%$ phosphotungstic acid solution for $10 \mathrm{~min}$; b) incubation in 1\% toluidine blue for $10 \mathrm{~min}$; c) incubation in $70 \%$ ethanol solution for 1 min to differentiate; d) staining with picric acid-orange mixture for $30 \mathrm{~s}$; e) staining in Ponceau mixture for $30 \mathrm{~min}$; f) slides were sealed with neutral resin after drying; the slides were washed with distilled water after each incubation step. The coloration of the stained sections was as follows: bone tissue was reddish brown; bone marrow stromal tissue or soft tissue was orange yellow; new bone and osteoid were blue; and cartilage and calcified cartilage were light blue.

\section{Hematoxylin and eosin $(\mathrm{H}+\mathrm{E})$ staining}

After biomechanical testing, the femoral condyle specimens were fixed in $4 \%$ paraformaldehyde at $4^{\circ} \mathrm{C}$ for $48 \mathrm{~h}$ and decalcified in $15 \%$ EDTA for 2 weeks. Then the specimens were routinely dehydrated for $24 \mathrm{~h}$ in an automatic dehydration machine, gradually taken through the clearing process, embedded in paraffin, sectioned, and dried for further staining. The section thickness was $4 \mu \mathrm{m}$. The staining protocol was as follows: 1) dewax the slides twice in xylene solution, 2 min each time; 2 ) rinse the slides twice with absolute ethanol to remove the residual xylene, 1 min each time; 3) sections were subsequently incubated in $95 \%$ and $80 \%$ ethanol solutions for 1 min each time; 4) the slides were then placed in a container and rinsed with slow-flowing water for $3 \mathrm{~min}$; 5) the slides were stained in Harris $\mathrm{H}+\mathrm{E}$ solution for $10 \mathrm{~min}$; 6) wash the slides under slow-flowing water for $3 \mathrm{~min}$ to completely remove the free stain; 7) differentiate the slides in $1 \%$ hydrochloric acid alcohol briefly; 8) the slides were incubated with $10 \%$ diluted ammonia briefly to stain the nuclei blue, then rinsed with slow-flowing water for $1 \mathrm{~min}$; 9) stain the slides in eosin solution for $1 \mathrm{~min}$ and wash in water again; 10) slides were incubated consecutively in $80 \%, 85 \%, 90 \%$, and $95 \%$ alcohol for $1 \mathrm{~min}$ in each gradient; 11 ) the slides were dehydrated twice in absolute ethanol, 1 min each time; 12) sections were cleared with xylene 3 times, 2 min each incubation; 13$)$ seal the slides with neutral resin.

\section{Histomorphometric analysis of the bone tissue}

The Leica QWin Standar V2.3 automatic image analyzing system (Germany) was used in histomorphometric analysis of the bone to determine the periprosthetic bone ingrowth and the bone formation. Eight images (magnification at $1.6 \times 10$ ) of Ponceau trichrome stained sections were collected from each coating group. Individual image regions in the area of 200 pixels around the prostheses were selected with image processing software; bone tissues in the selected regions including mature bone, cartilage, and osteoid were calculated using measurement tools of the software. Bone volume fractions (quotient of the bone tissue area in the selected region and the total area of the selected region) of different samples were calculated, and statistically compared.

\section{Biomechanical testing (push-out testing)}

Detection of the ultimate shear strength at the bone-prosthesis interface and analysis of the biomechanical properties of these prostheses were performed. Testing samples were collected from a circular area at the center of the prosthetic section with a radius of $1.5 \mathrm{~cm}$ and stuck on a predesigned mechanical testing platform with type II denture base resin. The vertical axis of prostheses 

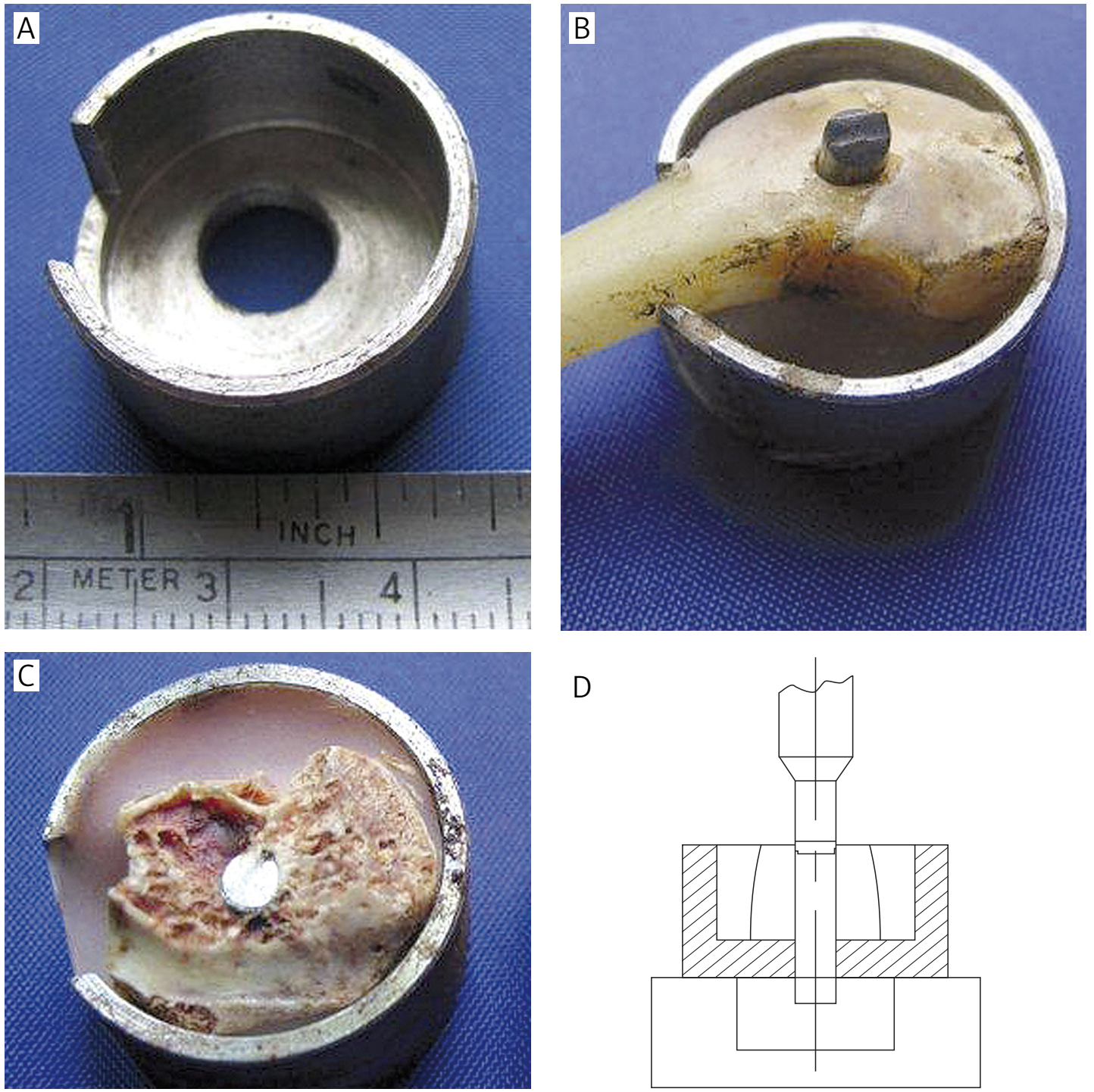

$\mathrm{D}$

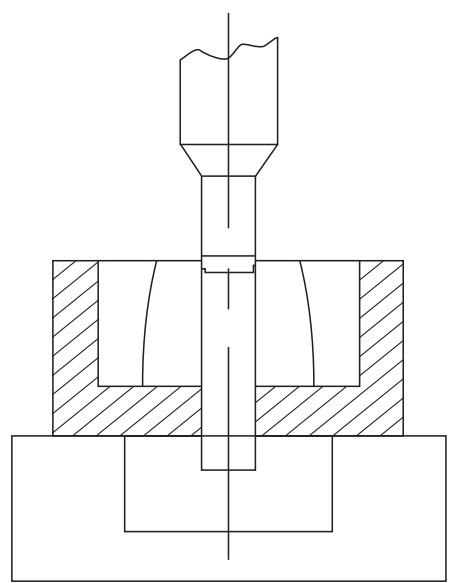

Figure 2. Platform for biomechanical testing and schematic diagram of testing process. A - Platform for push-out testing, which was designed according to the size of rabbit femoral condylar; B - demonstration experiment with bone specimen; $C$ - specimen was fixed on the platform; $D$ - schematic diagram of testing process; the prosthesis was pushed out from an eyelet at the bottom of the experimental platform by the pushing head

was kept perpendicular to the surface of the platform ( $\mathrm{a} \Phi=9 \mathrm{~mm}$ eyelet was made on the bottom of the mechanical testing platform to ensure that the prostheses could be pushed out from tissue during the push-out test). The computer-controlled INSTRON 6001 Universal Mechanical Testing System was used in this push-out testing to detect the shear effect on the prostheses with a loading speed of $0.5 \mathrm{~mm} / \mathrm{min}$ and an initial load of 0 . The value of ultimate shear strength was recorded when the implanted prosthesis started to move after continuously loading. The structure of the mechanical testing platform and the fixing effect of type II denture base resin are shown in Figures $2 \mathrm{~A}$-C. Figure $2 \mathrm{D}$ shows the conceptual diagram of the push-out test.

\section{The SEM observation and EDX analysis}

The prosthetic surface was scanned with a Hitachi S-3400N SEM, and EDX analysis was done simultaneously. The changes in surface morphology and element composition of the prostheses before and after the ultimate shear strength testing were examined. The condition of periprosthetic bone formation and the shear effect at the bone-prosthesis and the coating-substrate interfaces were also evaluated.

\section{Statistical analysis}

All data were statistically analyzed with SPSS 11.5 software and represented as $\bar{x} \pm \mathrm{s}$. Differences among groups were analyzed by ANOVA and LSD t-test. 


\section{Results}

\section{Adhesive strength between coating and prosthesis}

The appearance of two coatings has changed significantly after the scratch test. There are deep furrows and partial coating loss and peeling on the surfaces accompanied by substrate exposure (Figures $3 \mathrm{~A}$ and $\mathrm{B}$ ). The association curve between acoustic emission (AE) signal (due to interface peeling) and the load on diamond indenter $(P)$ indicates that HA coating peels when the load on diamond indenter is $11.18 \pm 1.28 \mathrm{~N}$ with more secondary peaks, whereas Ti coating starts to peel on the normal load of $17.69 \pm 1.66 \mathrm{~N}$ with relatively fewer secondary peaks. The threshold of normal load for HA coating to peel is statistically lower than that for $\mathrm{Ti}$ coating $(p<0.01$, Figure $3 C)$.

\section{Histological observation}

As shown in Figures $4 \mathrm{~A}$ and D, 4 weeks after the implantation, the bone formation around the HAcoated prostheses is significantly greater than that around the Ti-coated implants; new bone tissues bond closely and stably to these two coatings; the trabecular bones extend to the surrounding.

With prolonged time after the implantation, as shown in Figures 4 B, C, E, and F, the thickness of new bone tissues and the number of trabecular bones around the two coatings increase continuously.

Figures $5 \mathrm{~A}-\mathrm{F}$ and Table I reveal the volume of the periprosthetic bone around these two kinds of prostheses at different times after implantation. Figure $5 \mathrm{G}$ shows the comparison of bone volume fractions between two differently coated prostheses at different times after implantation; the results indicate that there are significant differences in periprosthetic bone volume fractions between $\mathrm{HA}$ - and $\mathrm{Ti}$ coated implants at the same time after surgery. Figure $5 \mathrm{H}$ shows the comparison of the bone volume fractions of HA-coated prostheses at different times after implantation; the results indicate that periprosthetic bone volume fractions of HA-coated prostheses at 15 weeks are significantly higher than those at 4 and 9 weeks after implantation. Histomorphometry results suggest that HA coating has an obvious advantage in periprosthetic osteogenesis, and the volume of bone around HA coatings increases continuously with time after implantation.

\section{The SEM observation and EDX analysis}

The SEM observation of the new tissues on the prosthetic surface shows the attachment of a thick layer of new bony tissues to the surface of HA-coated prostheses with irregular structures (Figure 6 A); a thin layer of bony tissues can be observed on the surface of the Ti-coated implant with regular, uni- form, and clear trabecular structure (Figure 6 B). As shown in Figure $6 \mathrm{C}$, EDX analysis reveals that the major component elements of the new tissues on surfaces of the two coatings are $\mathrm{C}, \mathrm{O}, \mathrm{Ca}$, and $\mathrm{P}$; this element composition is similar to that of bone tissue.

The results from histological observation, histomorphometry, SEM observation, and EDX analysis suggest that HA coatings have obvious advantages in osteogenesis and mineralization compared to Ti coatings at the early stage after prosthesis implantation.

\section{Push-out testing}

Table II shows the value of ultimate shear strength of these two kinds of prostheses at four, nine, and fifteen weeks after implantation. Figure $6 \mathrm{D}$ shows the ultimate shear strength of HA-coated prostheses at different times after implantation; the results indicate that the ultimate shear strength of both kinds of coated prostheses at 15 weeks is significantly higher than that at 4 weeks after implantation $(p<0.01)$, which consistent with the results from bone histomorphometry. However, the ultimate shear strength of HA-coated prostheses was much lower than that of $\mathrm{Ti}$ coated implants at the same stage after implantation ( $p<0.01$, Figure $6 \mathrm{E})$.

\section{Histological observation and EDX analysis after pull-out testing}

$\mathrm{H}+\mathrm{E}$ staining of the periprosthetic tissues after push-out testing on two kinds of prostheses shows that the periprosthetic trabecular bone is broken and the local trabecular bone near the prosthesis is damaged (Figures $7 \mathrm{~A}$ and $\mathrm{B}$ ). The gray cable-like clumps accumulates on the fractured bone surfaces after the push-out of HA-coated prostheses. However, no foreign residues can be seen on the fractured bone surfaces or in the surrounding medullary cavities of Ti-coated prostheses (Figures $7 \mathrm{C}$ and D). The EDX analysis of the prosthetic surface after the push-out test is shown in Figures $7 \mathrm{E}$ and $\mathrm{F}$; $\mathrm{Ti}$, $\mathrm{Al}$ and $\mathrm{V}$ elements can be detected on the surface of HA-coated prostheses after the push-out test, indicating the loss of HA coating and the exposure of substrate elements, whereas the element composition on the surface of Ti-coated implant shows no change. These results suggest that the structural integrity of HA coating is prone to damage under repeatedly changing loads and high shear stress; the low adhesive strength between coating and substrate is not conducive to the stability of $\mathrm{HA}$-coated prostheses in vivo.

\section{Discussion}

The stability of coated prostheses in vivo is affected by multiple factors, such as coating deco- 

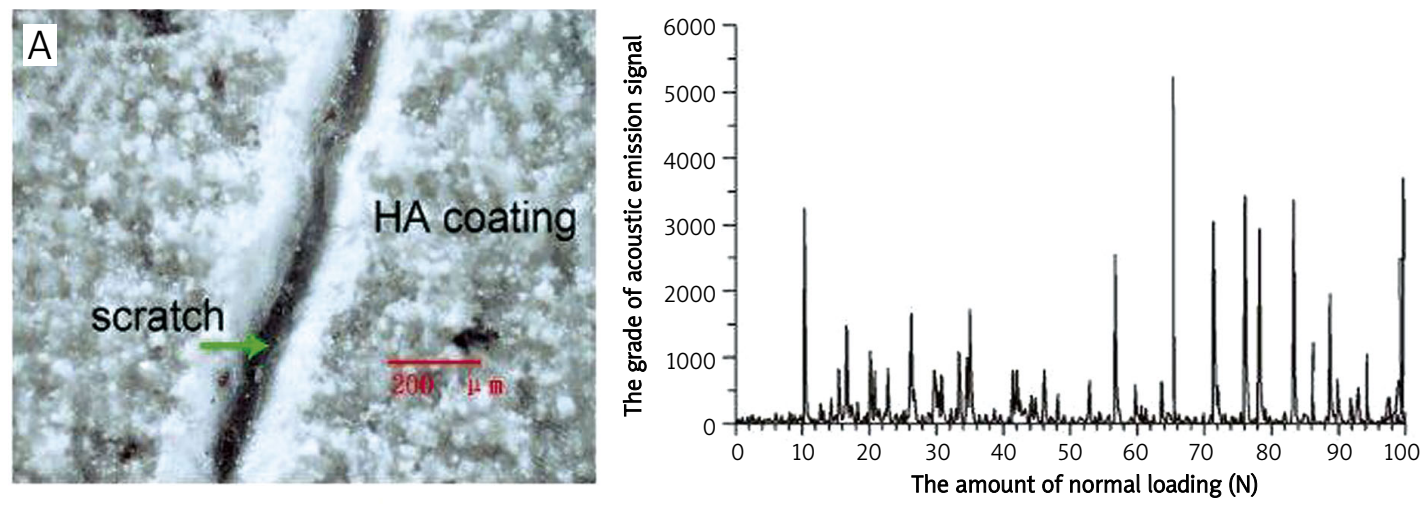
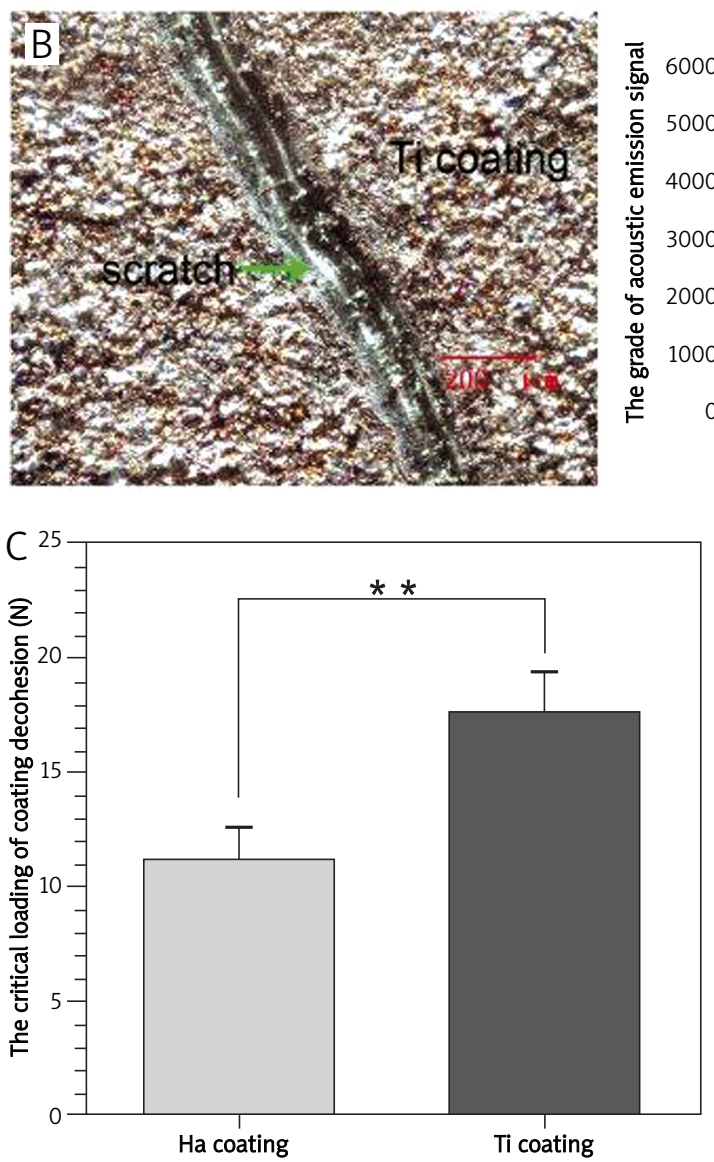

Figure 3. Morphological change of the two coatings after scratch testing and comparison of the adhesive strength of different coatings. A, B - Morphological change of $\mathrm{HA}$ and $\mathrm{Ti}$ coating after scratch testing and the related curve between the AE signals and the loads; $\mathrm{C}$ - threshold of the normal loading on different coatings

${ }^{\star *} p<0.01$ compared with Ti coating group

hesion, fracture, degradation, formation of coating particles, and tissue reaction and bone resorption around the prostheses. The adhesive strength at bone-coating and coating-substrate interfaces is the very important foundation for the stability of prostheses. If either of them cannot withstand the normal physiological load, loosening of the implanted prosthesis will occur [12-15].

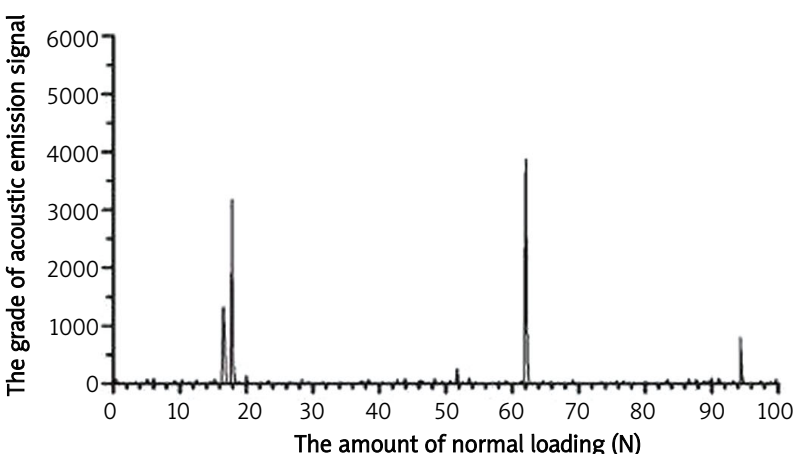

Table I. Bone volume fraction of the two coating groups at different times after implantation

\begin{tabular}{|lccc|}
\hline \multirow{2}{*}{ Groups } & \multicolumn{3}{c|}{ Bone volume fraction $(\%, n=8, \bar{x} \pm \mathrm{s})$} \\
\cline { 2 - 4 } & 4 weeks & 9 weeks & 15 weeks \\
\hline HA coating & $40.69 \pm 4.04$ & $54.05 \pm 3.90$ & $61.70 \pm 3.97$ \\
\hline Ti coating & $29.36 \pm 3.39$ & $36.35 \pm 3.03$ & $41.72 \pm 3.86$ \\
\hline
\end{tabular}

The scratch test is so far the major testing method for evaluation of the adhesive strength between coating and substrate [16-18]. A coating adhesion scratch tester was used to detect the threshold of normal loading on coatings when the coatings detached from the substrate. The determined normal loading threshold was then used to calculate the coating adhesive strength. When the tester head moves along the coating surfaces, the primary resistance comes from the coating friction, which also depends on the coating-substrate adhesive strength. With the increase in normal loading and parallel movement of the tester head, the resistance will come mainly from resistance at the coating-substrate interface, i.e., from the bonding force between coating and substrate. When normal loading reaches the threshold, the coating interface starts stripping and damaging; the acoustic emission (AE) signals are captured and recorded concurrently by a computer through the emission sensor system and form the first peak in the correlation curve between the AE signal and the load of the diamond tester head $(P)$. The tester head then encounters resistance that comes from the obsta- 

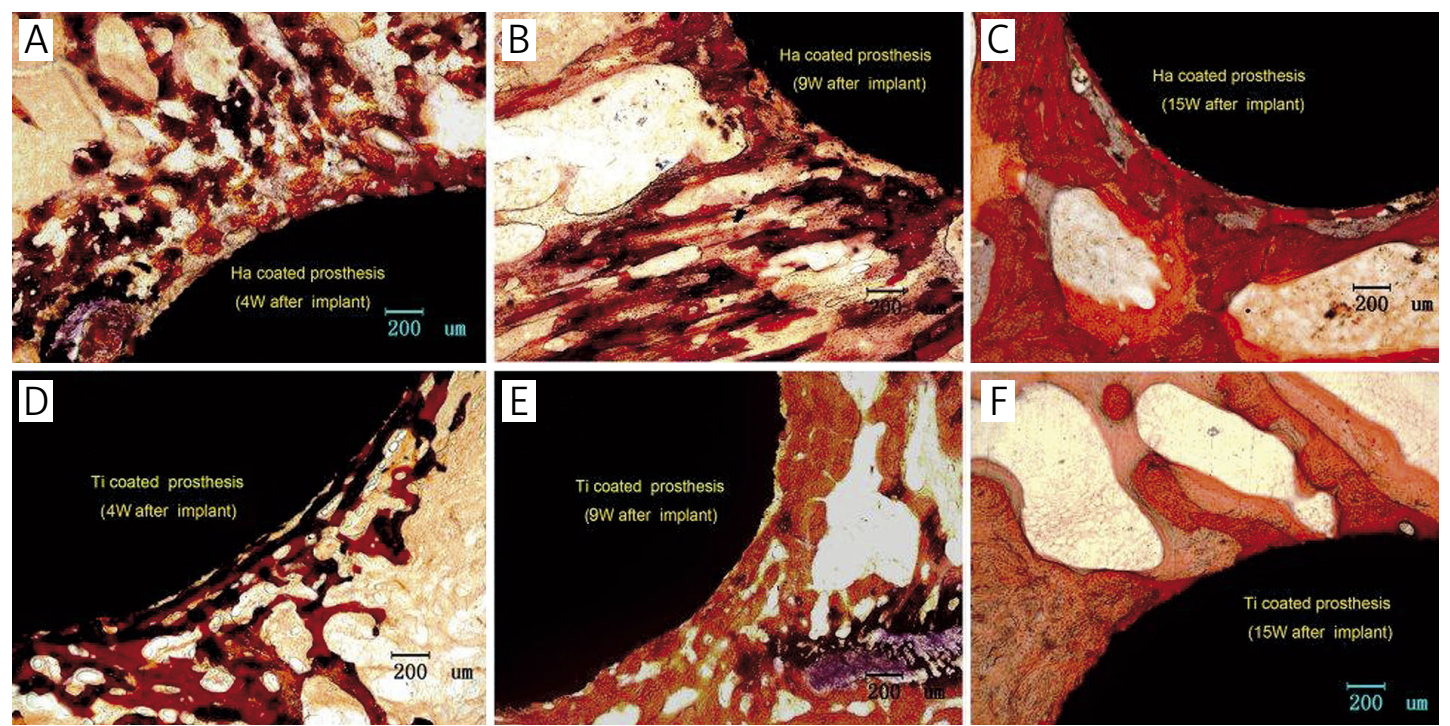

Figure 4. Improved Ponceau trichrome staining of histological section. A-C - show respectively the ingrowth bones of peri-prosthesis in HA coating group at 4, 9, and 15 weeks after implantation; D-F - show respectively the bone ingrowth of peri-prosthesis around Ti coatings at 4, 9, and 15 weeks after implantation

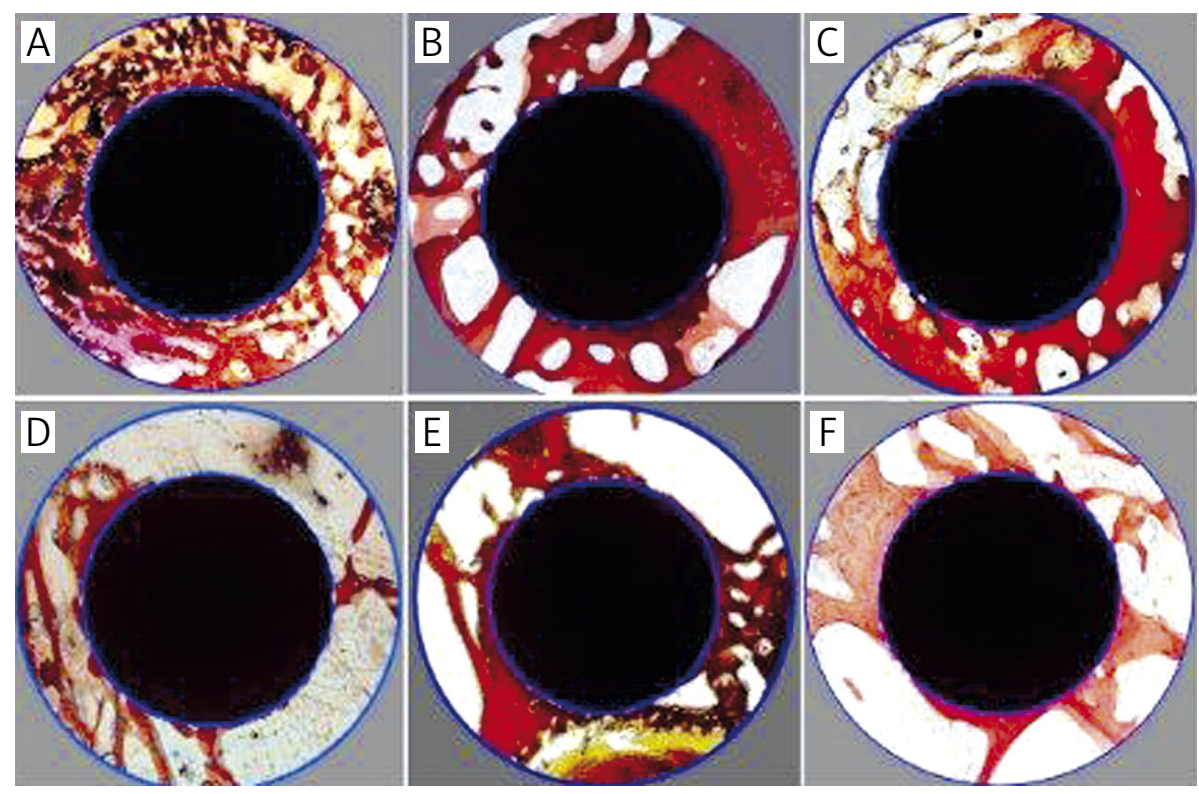

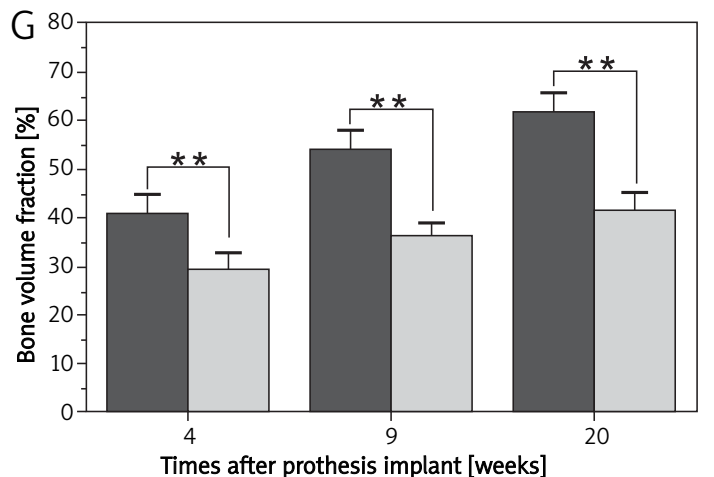

$\square$ HA coating

$$
\square \text { Ti coating }
$$

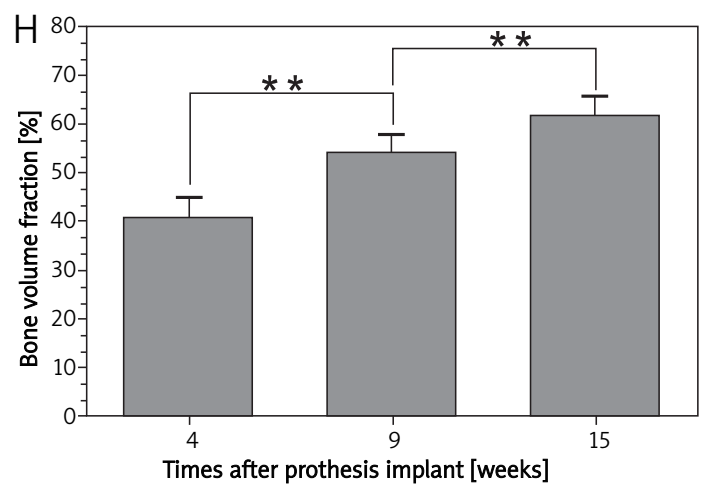

$\square$ HA coating

Figure 5. Comparison of bone volume fractions. A-C - Bone volume of peri-prosthesis around HA-coated prosthesis at different times after prosthesis implantation; $\mathrm{D}-\mathrm{F}$ - bone volume of peri-prosthesis around Ti-coated prosthesis at different times after prosthesis implantation; $\mathrm{G}$ - comparison of bone volume fractions between two differently coated prostheses at different times after implantation $\left({ }^{* *} p<0.01\right) ; \mathrm{H}-$ bone volume fractions of HA-coated prostheses at different times after implantation $\left({ }^{* *} p<0.01\right)$ 


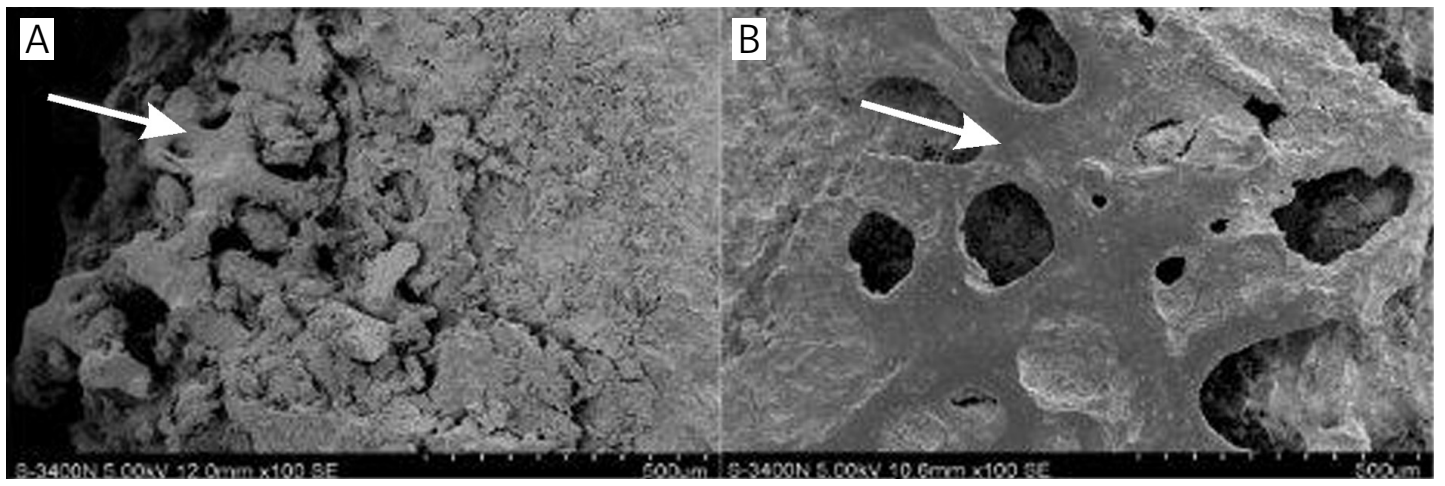

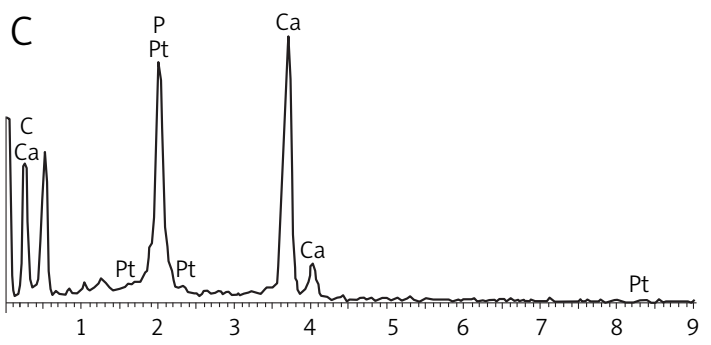

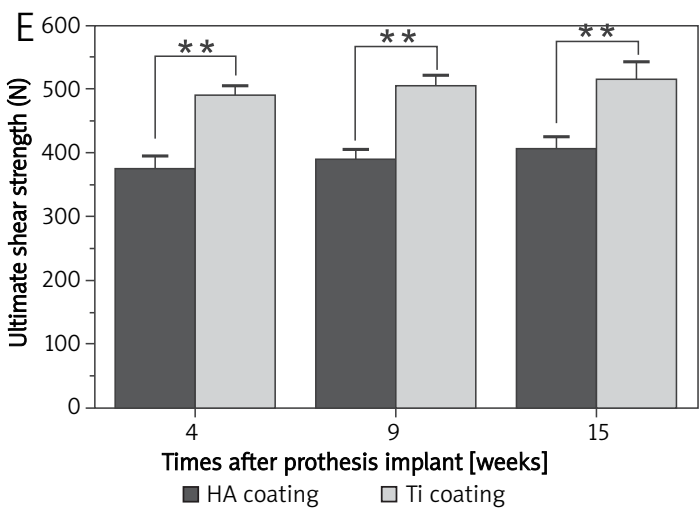

cle formed between the compressed, stripped coating and the interface; this resistance still reflects the adhesive strength between the coating and the substrate, and such interface damage manifests as a secondary peak in the imaging. Statistical analysis shows that the loading threshold for decohesion of HA coating is $11.18 \pm 1.28 \mathrm{~N}$ with more secondary peaks, whereas the loading threshold for $\mathrm{Ti}$ coating is $17.69 \pm 1.66 \mathrm{~N}$ with relatively fewer secondary peaks, implying that the adhesive strength

Table II. Ultimate shear strength of the two kinds of prosthesis at different times after prosthesis implantation

\begin{tabular}{|lccc|}
\hline \multirow{2}{*}{ Groups } & \multicolumn{3}{l}{ Ultimate shear strength $(N, n=6, \bar{x} \pm \mathrm{s})$} \\
\cline { 2 - 4 } & $\mathbf{4}$ weeks & 9 weeks & 15 weeks \\
\hline HA coating & $373.73 \pm 19.54$ & $390.88 \pm 15.08$ & $406.50 \pm 18.26$ \\
\hline Ti coating & $488.88 \pm 15.18$ & $504.63 \pm 17.06$ & $514.88 \pm 27.39$ \\
\hline
\end{tabular}

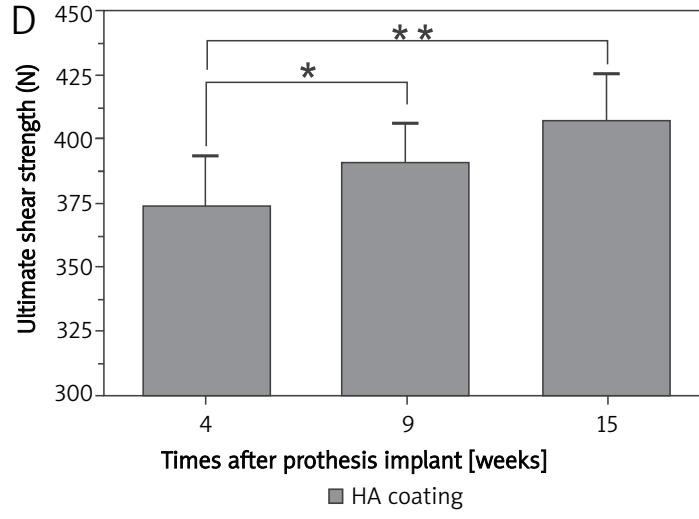

Figure 6. The image of SEM observation and EDX analysis and the comparison of ultimate shear strength on $\mathrm{HA}$ and $\mathrm{Ti}$ coated prostheses at different times after implantation. A - SEM observation of the surface tissues of HA-coated prostheses 4 weeks after implantation; B - SEM observation of the surface tissues of Ti-coated prostheses 4 weeks after implantation; C - EDX analysis image of the new tissues on surfaces of the two coatings; D - ultimate shear strength on HA-coated prostheses at different times after implantation ( ${ }^{* *} p<0.01,{ }^{*} p>0.05$ ); $\mathrm{E}$ - comparison of ultimate shear strength between two groups at the same stage after implantation $\left.{ }^{* *} p<0.01\right)$

of $\mathrm{HA}$ coating is significantly lower than that of $\mathrm{Ti}$ coating, and the HA coating is more easily damaged under a relatively strong external shear force.

Ultimate shear strength, the minimum load that is needed to separate the bone-prosthesis interface, is an effective parameter for testing the stability of implanted prostheses and is also an important biomechanical indicator that objectively reflects the adhesive strength between the prostheses and the periprosthetic new tissues as well as the prosthetic stability within the tissues. Higher ultimate shear strength reflects higher adhesive strength between prostheses and periprosthetic new tissues and between substrate and coating and indicates a better prosthetic stability within the tissues too. Therefore, the ultimate shear strength becomes a comprehensive indicator for testing the prosthetic stability in vivo $[19,20]$.

The results from histological observation, histomorphometric analysis, and biomechanical testing 

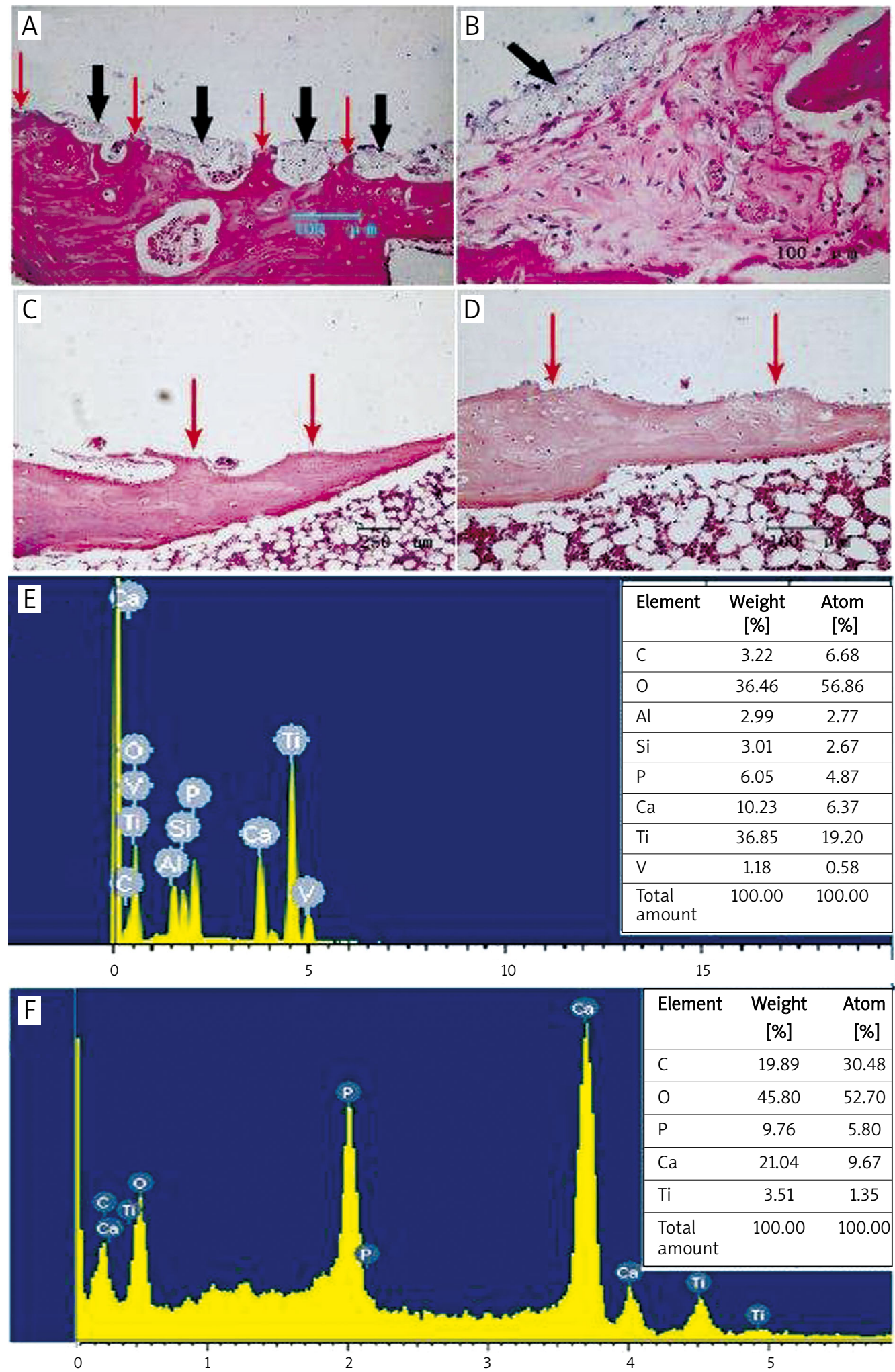

Figure 7. Histological observation and EDX analysis after pull-out testing. A, B $-H+E$ staining of the tissues surrounding HA-coated prosthesis after push-out test: fracture and defect of the periprosthetic trabecular bones (red arrows); discontinuous or continuous foreign residues at the tissue-prosthesis interface (black arrows); C, D $\mathrm{H}+\mathrm{E}$ staining of the tissues surrounding Ti-coated prosthesis after push-out test: fracture and defect of periprosthetic trabecular bones can be observed (red arrows); there are no foreign residues at the tissue-prosthesis interface; $\mathrm{E}$ - elements of $\mathrm{Ti}, \mathrm{Al}$, and $\mathrm{V}$ were detected from the HA-coated prosthetic surface after the push-out test, indicating substrate exposure; F - apart from Ga, P, and other bone component elements, Ti was the only element detected on the surface of Ti-coated prosthesis by EDX after the push-out test, suggesting a relatively intact structure of the Ti coating 
suggest that the bone formation on and around the prosthetic surface is an important factor that plays a positive role in stabilizing the implanted prostheses at the early stage after implantation. Although HA coating has an obvious advantage in osteogenesis, the ultimate shear strength of HA-coated prostheses was much lower than that of Ti coated implants ( $p<0.01$, Figure $6 \mathrm{E}$ ). These results indicate that the amount of osteogenesis around prostheses is not the only factor that determines the stability of HA-coated prostheses in vivo.

Further study through histological observation, SEM observation, and EDX analysis after push-out testing shows that the structural integrity of $\mathrm{HA}$ coating is prone to damage under repeatedly changing loads and high shear stress, and the low adhesive strength between coating and substrate is not conducive to the stability of HA-coated prostheses in vivo.

In conclusion, HA-coated prostheses are characterized by satisfactory attached bone growth and bone ingrowth on the porous coating surface, which exerts a positive effect on the biomechanical behavior and stability of coated prostheses at the early stage after implantation. The adhesive strength is an important factor for the stability of HA-coated prostheses in vivo. Due to the relatively low adhesive strength between coating and substrate, the structural integrity of HA coating is prone to damage under repeatedly changing loads and high shear stress, eventually affecting the long-term stability after prostheses implantation.

\section{Acknowledgments}

This work was funded by the Medical Science Research Foundation of Xijing Hospital (Grant Number XJZT09Z10). The coating materials were kindly supplied by Jinghang Institute of Biological Materials, which belongs to Beijing Institute of Aerospace Materials (China). The authors would like to thank Mr. Guogiang Ouyang and Prof. Huici Su for assisting in the experimental work, and Ms. Zhuoyi Yu for contributions to the preparation of testing materials.

Yonghong Duan, Shu Zhu and Fei Guo have contributed equally to this work.

\section{References}

1. Goller G, Oktar FN, Ozyegin LS, Kayali ES, Demirkesen E. Plasma-sprayed human bone-derived hydroxyapatite coatings: effective and reliable. Mater Lett 2004; 582: 599-604.

2. Kusakabe H, Sakamaki T, Nihei K, et al. Osseointegration of a hydroxyapatite-coated multilayered mesh stem. Biomaterials 2004; 2529: 57-69.

3. NCapello W, D'Antonio JA, Geesink RG, Feinberg JR, Naughton $\mathrm{M}$. Late remodeling around a proximally HAcoated tapered titanium femoral component. Clin Orthop Relat Res 2009; 4671: 55-65.
4. Dorairajan A, Reddy RM, Krikler S. Outcome of acetabular revision using an uncemented hydroxyapatite-coated component. J Arthroplasty 2005; 20: 209-18.

5. Chambers B, Clair SFS, Froimson MI. Hydroxyapatite-coated tapered cementless femoral components in total hip arthroplasty. J Arthroplasty 2007; 22 (4 Suppl 1): 71-4.

6. Cao N, Dong J, Wang Q, Ma Q, Xue C, Li M. An experimental bone defect healing with hydroxyapatite coating plasma sprayed on carbon/carbon composite implants. Surf Coat Technol 2010; doi:10.1016/j.surfcoat.2010.05.0081-7.

7. S $\varnothing$ balle K, Overgaard S. The current status of hydroxyapatite coating of prostheses. J Bone Joint Surg 1996; 78-B: 689-91.

8. Morscher EW, Hefti A, Aebi U. Severe osteolysis after thirdbody wear due to hydroxyapatite particles from acetabular cup coating. J Bone Joint Surg 1998; 80-B: 267-72.

9. Gross KA, Walsh W, Swarts E. Analysis of retrieved hydroxyapatite-coated hip prostheses. J Therm Spray Technol 2004; 13: 190-9.

10. Murty AN, Scott G, Freeman MAR. Hydroxyapatite-coated femoral components in total knee arthroplasty. J Arthroplasty 2003; 18: 844-51.

11. Wang F, Chen DY, Qiu XS, et al. Clinical analysis and prevention from coating shedding of uncemented hip prosthesis. China J Orthop Trauma 2009; 22: 413-6.

12. Nimb L, Gotfredsen K, Jensen JS. Mechanical failure of hydroxyapatite coated titanium and cobalt chromium molybdenum alloy implants: An animal study. Acta Orthop Belg 1993; 59: 333-8.

13. Faig-Martía J, Gil-Murb FJ. Hydroxyapatite coatings in prosthetic joints. Rev Esp Cir Ortop Traumatol 2008; 52: 113-20.

14. Morscher EW. Hydroxyapatite coating of prostheses. J Bone Joint Surg 1991; 73: 705-6.

15. Røkkum M, Reigstad A, Johansson CB. HA particles can be released from well-fixed HA-coated stems. Acta Orthop Scand 2002; 73: 298-306.

16. Xie Y, Hawthorne HM. A model for compressive coating stresses in the scratch adhesion test. Surf Coat Technol 2001; 14: 115-25.

17. Bull SJ, Berasetegui EG. An overview of the potential of quantitative coating adhesion measurement by scratch testing. Tribol Int 2006; 39: 99-114.

18. Valli J, Mäkelä U. Applications of the scratch test method for coating adhesion assessment. Wear 1987; 115: 215-22.

19. Chang Y, Kobayashi M, Li Z, Oka M, Nakamura T. Significance of peak value and duration of the interfacial shear loadin evaluation of the bone-implant interface. Clin Biomech 2003; 187: 73-9.

20. Castellani C, Lindtner RA, Hausbrandt P, et al. Boneimplant interface strength and osseointegration: Biodegradable magnesium alloy versus standard titanium control. Acta Biomater 2011; 7: 432-40. 